\title{
Modelling the Plant Uptake of Metals from Release Rates Obtained by the EUF Method
}

\author{
Manfred Sager (1)
}

Bioforschung Austria, Esslinger Hauptstrasse 134, A-1220 Vienna, Austria; m.sager@bioforschung.at

\begin{abstract}
In this study, soil dissolution kinetics were evaluated to predict the metal uptake of lettuce plants under varying conditions of fertilisation and metal pollution. Velocities and time dependencies of soil dissolution obtained by electro-ultrafiltration (EUF), which prevents back reaction, were modelled in three ways, obtained from suspensions in $0.002 \mathrm{M}$ DTPA at determined soil pH levels, for cases in which sampling versus time led to decreasing concentrations. The models yielded a maximum achievable concentration, a timespan needed for it to be reached, a slope, and an intercept of the respective fitted curves. Three geogenically metalliferous soil samples and one ambient soil sample, both as originals, fertilised with PK or soaked with a Cd-Ni-Pb solution, were used as solid samples. The resulting kinetic parameters were correlated with the amounts absorbed by lettuce plants grown with these substrates in pot experiments, which yielded fairly good correlations with $\mathrm{Zn}$, but also with $\mathrm{Li}$ and $\mathrm{Sr}$, as well as $\mathrm{Ni}$ and $\mathrm{Pb}$, mainly because of differences due to the addition of a metallic salt solution. Plant growth was hardly influenced by the additions.
\end{abstract}

Keywords: EUF; soil dissolution kinetics; lettuce; metal uptake

\section{Introduction}

The uptake of several elements by healthy green plants from soil depends on the Citation: Sager, M. Modelling the Plant Uptake of Metals from Release Rates Obtained by the EUF Method. Plants 2022, 11, 85. https://doi.org/ $10.3390 /$ plants 11010085

Academic Editor: Paramsothy Jeyakumar

Received: 30 November 2021

Accepted: 27 December 2021

Published: 28 December 2021

Publisher's Note: MDPI stays neutral with regard to jurisdictional claims in published maps and institutional affiliations.

Copyright: () 2021 by the author. Licensee MDPI, Basel, Switzerland. This article is an open access article distributed under the terms and conditions of the Creative Commons Attribution (CC BY) license (https:// creativecommons.org/licenses/by/ $4.0 /)$. release rate and transport process in the soil, as well as on plant need, the number of receptors, and excretion of plant roots [1,2]. Though some legal thresholds to use arable soils for crop production refer to aqua regia digests (e.g., for $\mathrm{Pb}$ and $\mathrm{Cd}$ ), it has been known for a long time that the total contents present in the soil are not entirely available to plants, due to various release rates, transport processes to the roots, and plant needs. This might be particularly problematic for soils developed on metalliferous rocks [3]. In agriculture, the plant-available "fraction" of a given substrate assumes how much a root can take within one growing season. For reasons of simplicity and costs of investigation, this study sought to imitate this kinetic process by a single-step, partial extraction with organic acids and/or complexants, which release more easily mobilizable elements to the soil solution, irrespective of the chemical speciation of the solid phase of the soil. In sequential leaching, steps of decreasing mobility and plant availability due to dissolution and desorption reactions were obtained, which could be assigned to different solid soil phases, such as exchangeable, carbonate, paedogenic oxide, humic, sulphide, or silicate phases. The availability from these phases varies due to different environmental conditions and time scales. Uncertainties derive from desorption from non-dissolved phases, resorptions at the remaining solid, deviations of chemical properties of the target ion (e.g., no carbonates formed, oxides not easily soluble in acid), or the presence of phases not taken into account by the model [4,5]. Multi-element methods such as ICP-OES permit the extension of element determinations in a solution to much more dissolved elements than originally verified by test minerals. They were thus operationally defined.

In this study, the kinetic process of plant uptake was modelled by soil dissolution kinetics. Contrary to selective leaching methods, which approach mobilisations in a snapshot, kinetic methods simulate desorption from the solid soil particles. This assumes that plant 
uptake is faster than dissolution, and the uptake is independent of plant needs and uptake exclusion metabolism.

Modelling of release rates versus time permits interpretations of dissolution mechanisms, which may differ among items released from the same substrate [6]. If the release rate is controlled by transport only, it is proportional to the distance from equilibrium, as in the case of film diffusion, intra-particle diffusion, and pore diffusion. Many dissolution processes of geochemical relevance, however, are governed by surface properties, such as crystallisation, impurities, or sorbed species [7].

\section{Material and Methods}

\subsection{Soil Samples}

As test samples, three arable soils from geogenically metalliferous sites and one ambient soil sample from the Austrian Province of Styria were selected (Table 1). The metalliferous soils had developed above sulfidic ore veins containing As, $\mathrm{Cd}, \mathrm{Cu}, \mathrm{Pb}$, and $\mathrm{Zn}$, but baryte, quartz, pyromorphite, cerussite, and malachite $[3,8]$ have also been occasionally found.

Table 1. Test soils prior to the pot experiment.

\begin{tabular}{|c|c|c|c|c|c|c|c|c|c|c|c|}
\hline Location & $\mathrm{pH}$ & Sand \% & Silt \% & Clay \% & $\begin{array}{c}\text { C-org } \\
\mathrm{mg} / \mathrm{kg}\end{array}$ & $\begin{array}{c}\mathrm{Pb} \\
\mathrm{mg} / \mathrm{kg}\end{array}$ & $\begin{array}{c}\mathrm{Ni} \\
\mathrm{mg} / \mathrm{kg}\end{array}$ & $\begin{array}{c}\mathrm{Cd} \\
\mathrm{mg} / \mathrm{kg}\end{array}$ & $\begin{array}{c}\mathrm{Cu} \\
\mathrm{mg} / \mathrm{kg}\end{array}$ & $\begin{array}{c}\mathrm{Cr} \\
\mathrm{mg} / \mathrm{kg}\end{array}$ & $\begin{array}{c}\mathrm{As} \\
\mathrm{mg} / \mathrm{kg}\end{array}$ \\
\hline Rabenstein & 6.9 & 46 & 41 & 13 & 2.8 & 340 & 42 & 1.8 & 47 & 27 & 19 \\
\hline Arzwaldgraben & 7.4 & 26 & 64 & 10 & 5.4 & 800 & 51 & 2.6 & 45 & 75 & 12 \\
\hline Zeltweg & 6.2 & 30 & 62 & 8 & 4.1 & 130 & 43 & 0.24 & 44 & 58 & 35 \\
\hline Kraubath & 7.3 & 44 & 47 & 9 & 4.6 & 21 & 24 & $<0.2$ & 36 & 26 & 8 \\
\hline
\end{tabular}

The soil samples were obtained from at least 25 individual cores at 5-30 cm depth, merged on site, dried at $40^{\circ}$, and sieved minor $2 \mathrm{~mm}$ [9]. The soil pH was determined in $0.01 \mathrm{M} \mathrm{CaCl}_{2}$ [10], the clay-silt-sand distribution by the pipette method [11], the total organic carbon (C-org) by combustion [12], and the pseudo-total contents by inductively coupled plasma emission spectrometry after digestion with aqua regia (Table 1). The statistical validity of these standard methods was annually tested by ring tests run by the ALVA organisation, in which we participated. With respect to precisions, standard deviations of the parameters clay-silt-sand were within 1.5-2.8\% absolute, organic carbon within $0.08-0.21 \%$ absolute, and soil $\mathrm{pH}$ within $0.08-0.14$ units. For $\mathrm{As}, \mathrm{Cr}, \mathrm{Ni}, \mathrm{Pb}$, and $\mathrm{Zn}$ in aqua regia, precisions of \pm 6 to $13 \%$ of the value were achieved, and for $\mathrm{Cd}, \pm 13$ to $20 \%$ (unpublished internal data) was obtained.

For the pot experiments, these soils, as well as the resulting soils after the addition of a $\mathrm{Cd}-\mathrm{Ni}-\mathrm{Pb}$ solution or $\mathrm{PK}$ fertiliser, were used, with three replicates each.

\subsection{Test Plants}

As test plants, lettuce (Lactuca sativa) was chosen, which is known as a rapidly growing and universally accumulating species [13]. Three lettuce seedlings were randomly planted at Kick-Brauckmann pots containing $8 \mathrm{~kg}$ of dried soil $(\leq 20 \mathrm{~mm})$, which were placed randomly in a foliar-covered greenhouse, and watered each 3rd day. Then, 10 days after planting, the samples marked as "PK" in Table 2 received an addition of $225 \mathrm{mg} / \mathrm{kg} \mathrm{P}+128 \mathrm{mg} / \mathrm{kg} \mathrm{K}$ from a combination of superphosphate and potassium chloride. At the same time, the samples marked as "metal" received an addition of $0.75 \mathrm{mg} / \mathrm{kg}$ $\mathrm{Cd}+94 \mathrm{mg} / \mathrm{kg} \mathrm{Ni}+94 \mathrm{mg} / \mathrm{kg}$ Pb with respect to the test substrate, from $15 \mathrm{~mL}$ of a mixed solution containing $0.384 \mathrm{~g} \mathrm{Cd}\left(\right.$ as $\left.\mathrm{Cd}\left(\mathrm{NO}_{3}\right)_{2} \cdot 4 \mathrm{H}_{2} \mathrm{O}\right), 48 \mathrm{~g} \mathrm{Ni}\left(\right.$ as $\left.\mathrm{NiSO}_{4} \cdot 6 \mathrm{H}_{2} \mathrm{O}\right)$, and $48 \mathrm{~g} \mathrm{~Pb}$ (as $\mathrm{Pb}$ (acetate $)_{2} \cdot 3 \mathrm{H}_{2} \mathrm{O}$ ) in $1 \mathrm{~L}$, to test the uptake potential of lettuce for those metals. After 40 days of growth, roots and shoots were harvested separately to obtain yields in terms of wet weight. The samples were dried at $65^{\circ} \mathrm{C}$ for $72 \mathrm{~h}$, milled, and analysed for total metal contents by simultaneous multi-element analysis by ICP-OES (PerkinElmer Optima 
3000XL) after digestion with nitric acid in closed pressure vessels by microwave-assisted heating.

Table 2. Modelling the released concentrations from accumulated data.

\begin{tabular}{ccc}
\hline Kinetic Model. & $\mathbf{f}(\mathbf{x})$ & Release Rate $=\mathbf{d y} / \mathbf{d t}$ \\
\hline Linear equation & $\mathrm{y}=\mathrm{b}+\mathrm{at}$ & $\mathrm{dy} / \mathrm{dt}=\mathrm{a}$ \\
Elovich equation & $\mathrm{y}=\mathrm{b}+\mathrm{a} \ln (\mathrm{t})$ & $\mathrm{dy} / \mathrm{dt}=\mathrm{a} / \mathrm{t}$ \\
Weber-Morris equation & $\mathrm{y}=\mathrm{b}+\mathrm{a} \sqrt{\mathrm{t}}$ & $\mathrm{dy} / \mathrm{dt}=\mathrm{a} / \sqrt{\mathrm{t}}$ \\
Power equation & $\ln (\mathrm{y})=\mathrm{b}+\mathrm{a} \ln (\mathrm{t})$ & $\mathrm{dy} / \mathrm{dt}=\mathrm{a} / \mathrm{t} \cdot(\mathrm{b}+\mathrm{a} \ln (\mathrm{t}))$ \\
\hline
\end{tabular}

$\mathrm{y}=$ desorbed number of concentrations; $\mathrm{t}=$ time

\subsection{EUF Procedure}

After harvest, soil samples from the pot experiments were examined at the Justus Liebig Laboratory of Südzucker AG Company at Rain (Germany) by using a modified electro-ultrafiltration (EUF) method.

Electro-ultrafiltration (EUF) is a quick method originally designed to determine available plant nutrients and respective fertilisation needs from aqueous soil suspensions, such as nitrate, total dissolved nitrogen, phosphate, and potassium [14,15]. The slurry sample is put into a reaction chamber with electrodes and semipermeable membranes opposite each other, backed by solute-filled chambers for sampling (Figure 1). When the voltage and magnetic stirring are turned on, released ions move towards the respective electrode across the membrane and can be sampled from the anode and cathode chamber without further filtration or centrifugation. The migration velocity is proportional to the electric field strength and inverse to the friction in the fluid; it increases with increasing concentration and temperature. Charged colloids move much slower and hardly penetrate the membranes, which resembles the conditions at the root surface. As liberated ions are rapidly removed from the solid surfaces by the electric field, the back reaction is prevented; thus, the release rate is rapidly experimentally accessible. Variations in EUF conditions are possible within the same reaction cell, to obtain release rates under different conditions. Contrary to release rates obtained by EUF, batch methods target dissolution equilibria.

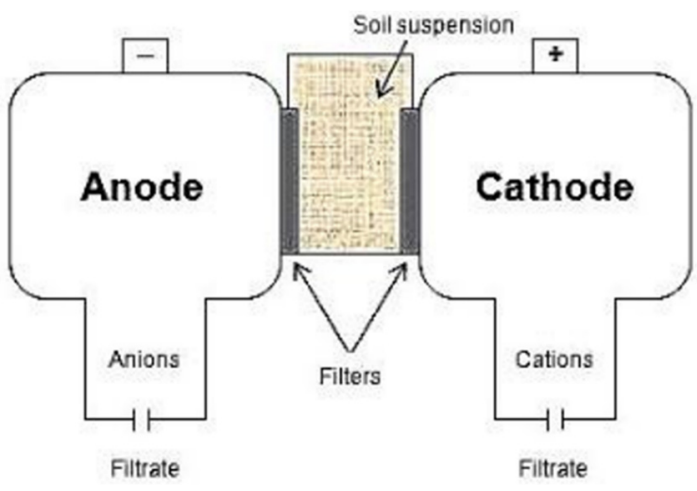

Figure 1. The electro-ultrafiltration (EUF) chamber.

The experimental EUF parameters had been developed originally to estimate the immediate nutrient availability for ryegrass (lolium perenne) and sugar beet at $200 \mathrm{~V} / 20^{\circ} \mathrm{C} / 30 \mathrm{~min}$, which was considered here for practical reasons. Due to $\mathrm{H}^{+}$consumption at the cathode, the cathode space becomes alkaline, and due to $\mathrm{OH}^{-}$consumption at the anode, the anode space becomes acidic. As many hydroxides and hydrated oxides are hardly soluble in the alkaline cathode extract, a complexing agent needs to be added, to determine metal cations (except $\mathrm{Na}, \mathrm{K}$ ). In order to avoid hydroxide precipitations in the cathode chamber, a suspension of $5.00 \mathrm{~g}$ of sample in $50 \mathrm{~mL} 0.002 \mathrm{M} \mathrm{DTPA}\left(\mathrm{C}_{14} \mathrm{H}_{23} \mathrm{~N}_{3} \mathrm{O}_{10}\right)$ aqueous solution (also known as Merck Titriplex V) was introduced into the EUF chamber system, and five separate fractions were collected every five minutes (in a total of $25 \mathrm{~min}$ ), 
applying $200 \mathrm{~V}$ and maximum $15 \mathrm{~mA}$ at ambient temperature [16]. After each extraction, the released ions were analysed by ICP-OES, and the concentration in solution times $\mathrm{ml}$ filtrate yielded the $\mathrm{mg} / \mathrm{kg}$ soil (listed in Table 3).

Table 3. Released amounts after 5, 10, 15, 20, and 25 min of electro-ultrafiltration in 0.002 M DTPA.

\begin{tabular}{|c|c|c|c|c|c|c|c|c|c|c|c|c|c|c|c|c|}
\hline Al & As & $\mathrm{Be}$ & $\mathrm{Cd}$ & Co & $\mathrm{Cr}$ & $\mathrm{Cu}$ & $\mathrm{Fe}$ & $\mathbf{L i}$ & Mn & $\mathrm{Ni}$ & $\mathbf{P b}$ & $\mathrm{Sb}$ & Sr & $\mathrm{Ti}$ & $\mathbf{V}$ & $\mathrm{Zn}$ \\
\hline \multicolumn{17}{|c|}{ Rabenstein } \\
\hline 5.42 & 0.349 & 0.092 & 0.311 & 0.364 & 0.163 & 3.35 & 25.57 & 0.0466 & 36.68 & 1.34 & 21.62 & 0.121 & 0.912 & 0.082 & 0.096 & 17.34 \\
\hline 9.93 & 0.232 & 0.078 & 0.203 & 0.588 & 0.136 & 2.01 & 36.24 & 0.0298 & 45.72 & 1.29 & 23.83 & 0.118 & 0.847 & 0.078 & 0.056 & 14.62 \\
\hline 11.97 & 0.141 & 0.072 & 0.120 & 0.652 & 0.114 & 0.80 & 34.05 & 0.0194 & 41.16 & 0.75 & 18.44 & 0.108 & 0.641 & 0.098 & 0.048 & 9.03 \\
\hline 14.37 & 0.102 & 0.074 & 0.085 & 0.711 & 0.117 & 0.47 & 34.06 & 0.0148 & 39.93 & 0.69 & 16.56 & 0.111 & 0.544 & 0.090 & 0.044 & 6.53 \\
\hline 15.72 & 0.078 & 0.073 & 0.061 & 0.701 & 0.114 & 0.18 & 32.67 & 0.0119 & 37.13 & 0.53 & 13.67 & 0.110 & 0.475 & 0.096 & 0.042 & 4.87 \\
\hline \multicolumn{17}{|c|}{ Rabenstein + PK } \\
\hline 3.58 & 0.253 & 0.074 & 0.222 & 0.271 & 0.063 & 0.491 & 19.35 & 0.0271 & 31.54 & 0.89 & 18.69 & 0.112 & 0.865 & 0.009 & 0.027 & 14.93 \\
\hline 7.44 & 0.194 & 0.076 & 0.168 & 0.471 & 0.079 & 0.327 & 29.44 & 0.0238 & 39.28 & 0.64 & 21.46 & 0.115 & 0.834 & 0.033 & 0.050 & 13.36 \\
\hline 9.97 & 0.132 & 0.074 & 0.112 & 0.593 & 0.083 & 0.017 & 31.15 & 0.0176 & 39.20 & 0.50 & 19.10 & 0.112 & 0.668 & 0.054 & 0.039 & 9.35 \\
\hline 11.70 & 0.095 & 0.073 & 0.076 & 0.642 & 0.083 & 0.016 & 30.45 & 0.0131 & 37.46 & 0.40 & 16.49 & 0.110 & 0.558 & 0.065 & 0.033 & 6.63 \\
\hline 12.91 & 0.069 & 0.073 & 0.053 & 0.638 & 0.083 & 0.016 & 29.53 & 0.0104 & 35.34 & 0.30 & 13.69 & 0.109 & 0.477 & 0.069 & 0.030 & 4.91 \\
\hline \multicolumn{17}{|c|}{ Rabenstein + metal } \\
\hline 4.11 & 0.736 & 0.084 & 0.704 & 0.306 & 0.099 & 1.01 & 26.75 & 0.0275 & 29.16 & 42.75 & 18.93 & 0.116 & 0.910 & 0.045 & 0.037 & 14.73 \\
\hline 8.62 & 0.560 & 0.076 & 0.529 & 0.535 & 0.105 & 0.88 & 39.97 & 0.0143 & 36.24 & 42.04 & 19.81 & 0.115 & 0.844 & 0.044 & 0.031 & 12.96 \\
\hline 11.23 & 0.359 & 0.074 & 0.331 & 0.647 & 0.104 & 0.47 & 40.15 & 0.0110 & 35.47 & 29.27 & 17.33 & 0.112 & 0.813 & 0.061 & 0.035 & 8.63 \\
\hline 13.04 & 0.239 & 0.073 & 0.219 & 0.685 & 0.108 & 0.15 & 38.57 & 0.0084 & 33.67 & 20.02 & 14.27 & 0.110 & 0.576 & 0.087 & 0.030 & 5.93 \\
\hline 13.85 & 0.167 & 0.072 & 0.147 & 0.654 & 0.110 & 0.02 & 35.61 & 0.0068 & 30.88 & 13.79 & 11.56 & 0.108 & 0.480 & 0.080 & 0.029 & 4.26 \\
\hline \multicolumn{17}{|c|}{ Arzwaldgraben } \\
\hline 2.503 & 0.425 & 0.074 & 0.390 & 0.258 & 0.117 & 1.102 & 22.36 & 0.041 & 31.32 & 0.790 & 64.21 & 0.108 & 1.712 & 0.016 & 0.064 & 21.16 \\
\hline 2.964 & 0.326 & 0.068 & 0.295 & 0.469 & 0.118 & 1.127 & 34.48 & 0.032 & 44.01 & 0.780 & 74.43 & 0.105 & 1.535 & 0.013 & 0.079 & 18.48 \\
\hline 3.306 & 0.213 & 0.064 & 0.189 & 0.625 & 0.111 & 0.896 & 35.78 & 0.023 & 45.57 & 0.525 & 61.28 & 0.099 & 1.208 & 0.021 & 0.079 & 13.20 \\
\hline 3.911 & 0.153 & 0.063 & 0.132 & 0.696 & 0.112 & 0.380 & 36.54 & 0.017 & 45.09 & 0.433 & 51.20 & 0.098 & 1.042 & 0.033 & 0.080 & 10.48 \\
\hline 4.475 & 0.110 & 0.060 & 0.092 & 0.663 & 0.108 & 0.233 & 34.97 & 0.014 & 39.71 & 0.370 & 40.80 & 0.093 & 0.893 & 0.042 & 0.076 & 7.86 \\
\hline \multicolumn{17}{|c|}{ Arzwaldgraben + PK } \\
\hline 3.942 & 0.378 & 0.078 & 0.343 & 0.240 & 0.100 & 1.567 & 24.09 & 0.045 & 23.48 & 0.658 & 61.41 & 0.112 & 1.547 & 0.018 & 0.098 & 18.87 \\
\hline 4.968 & 0.293 & 0.069 & 0.263 & 0.521 & 0.098 & 1.509 & 35.06 & 0.033 & 42.75 & 0.739 & 68.02 & 0.106 & 1.341 & 0.063 & 0.105 & 15.93 \\
\hline 5.560 & 0.199 & 0.065 & 0.175 & 0.736 & 0.098 & 0.903 & 37.46 & 0.023 & 49.80 & 0.592 & 56.00 & 0.100 & 1.091 & 0.054 & 0.104 & 12.13 \\
\hline 6.309 & 0.143 & 0.063 & 0.124 & 0.802 & 0.103 & 0.541 & 37.76 & 0.018 & 49.14 & 0.523 & 45.30 & 0.097 & 0.917 & 0.071 & 0.102 & 9.03 \\
\hline 7.177 & 0.110 & 0.061 & 0.093 & 0.768 & 0.106 & 0.342 & 37.51 & 0.014 & 44.26 & 0.458 & 37.14 & 0.094 & 0.807 & 0.085 & 0.096 & 7.03 \\
\hline \multicolumn{17}{|c|}{ Arzwaldgraben + metal } \\
\hline 2.786 & 1.542 & 0.072 & 1.393 & 0.224 & 0.096 & 1.454 & 20.28 & 0.040 & 28.15 & 54.28 & 63.18 & 0.108 & 1.477 & 0.005 & 0.076 & 15.26 \\
\hline 3.795 & 1.147 & 0.068 & 1.021 & 0.468 & 0.111 & 1.612 & 32.51 & 0.032 & 39.69 & 57.51 & 71.83 & 0.106 & 1.390 & 0.006 & 0.105 & 14.05 \\
\hline 4.303 & 0.730 & 0.064 & 0.696 & 0.652 & 0.106 & 1.119 & 35.41 & 0.022 & 42.84 & 42.88 & 59.46 & 0.100 & 1.012 & 0.023 & 0.096 & 10.26 \\
\hline 4.842 & 0.491 & 0.062 & 0.463 & 0.714 & 0.106 & 0.627 & 35.57 & 0.016 & 41.78 & 32.79 & 47.85 & 0.096 & 0.848 & 0.031 & 0.084 & 7.46 \\
\hline 5.512 & 0.351 & 0.061 & 0.327 & 0.694 & 0.108 & 0.354 & 36.43 & 0.013 & 39.27 & 24.99 & 38.84 & 0.094 & 0.745 & 0.037 & 0.081 & 5.73 \\
\hline \multicolumn{17}{|c|}{ Zeltweg } \\
\hline 11.63 & 0.078 & 0.084 & 0.058 & 0.119 & 0.119 & 1.901 & 27.36 & 0.045 & 9.57 & 0.48 & 8.38 & 0.122 & 1.081 & 0.168 & 0.235 & 1.020 \\
\hline 18.20 & 0.038 & 0.073 & 0.022 & 0.183 & 0.106 & 1.692 & 38.68 & 0.034 & 14.52 & 0.81 & 7.95 & 0.119 & 0.949 & 0.255 & 0.299 & 0.991 \\
\hline 20.80 & 0.024 & 0.072 & 0.009 & 0.214 & 0.111 & 1.115 & 39.90 & 0.027 & 13.78 & 0.68 & 5.87 & 0.116 & 0.781 & 0.298 & 0.284 & 0.598 \\
\hline 22.68 & 0.016 & 0.072 & 0.003 & 0.219 & 0.114 & 0.780 & 39.33 & 0.021 & 12.47 & 0.62 & 4.46 & 0.117 & 0.644 & 0.319 & 0.261 & 0.418 \\
\hline 23.18 & 0.013 & 0.071 & 0.000 & 0.206 & 0.113 & 0.503 & 36.87 & 0.017 & 11.03 & 0.64 & 3.35 & 0.113 & 0.527 & 0.316 & 0.226 & 0.354 \\
\hline & & & & & & & & ltweg + & & & & & & & & \\
\hline 11.91 & 0.096 & 0.091 & 0.075 & 0.150 & 0.147 & 1.702 & 32.84 & 0.040 & 15.88 & 0.48 & 9.34 & 0.121 & 1.053 & 0.192 & 0.222 & 0.752 \\
\hline 18.08 & 0.037 & 0.074 & 0.020 & 0.147 & 0.108 & 1.427 & 42.61 & 0.023 & 13.92 & 0.39 & 8.41 & 0.115 & 0.895 & 0.262 & 0.253 & 0.259 \\
\hline 21.29 & 0.023 & 0.074 & 0.007 & 0.171 & 0.115 & 0.995 & 43.17 & 0.019 & 12.14 & 0.28 & 6.43 & 0.115 & 0.750 & 0.310 & 0.248 & 0.106 \\
\hline 22.74 & 0.017 & 0.073 & 0.001 & 0.176 & 0.114 & 0.594 & 40.76 & 0.015 & 10.71 & 0.20 & 4.83 & 0.113 & 0.614 & 0.319 & 0.233 & 0.003 \\
\hline 23.12 & 0.013 & 0.072 & 0.000 & 0.166 & 0.117 & 0.377 & 38.00 & 0.013 & 9.54 & 0.15 & 3.62 & 0.110 & 0.517 & 0.316 & 0.204 & 0.043 \\
\hline & & & & & & & & weg $+\mathrm{m}$ & & & & & & & & \\
\hline 10.42 & 0.288 & 0.075 & 0.252 & 0.090 & 0.110 & 1.264 & 28.23 & 0.027 & 12.26 & 13.43 & 8.67 & 0.117 & 1.059 & 0.160 & 0.227 & 0.723 \\
\hline 16.70 & 0.201 & 0.077 & 0.174 & 0.148 & 0.136 & 1.112 & 41.51 & 0.026 & 13.42 & 12.72 & 8.27 & 0.119 & 0.939 & 0.285 & 0.322 & 0.464 \\
\hline 19.60 & 0.125 & 0.077 & 0.103 & 0.170 & 0.135 & 0.664 & 42.84 & 0.021 & 11.85 & 8.98 & 6.25 & 0.118 & 0.785 & 0.349 & 0.301 & 0.238 \\
\hline 21.22 & 0.082 & 0.077 & 0.062 & 0.172 & 0.136 & 0.295 & 41.34 & 0.017 & 10.47 & 6.25 & 4.60 & 0.116 & 0.641 & 0.362 & 0.264 & 0.111 \\
\hline 22.24 & 0.055 & 0.076 & 0.036 & 0.164 & 0.132 & 0.077 & 39.12 & 0.014 & 9.42 & 4.45 & 3.50 & 0.115 & 0.537 & 0.358 & 0.232 & 0.071 \\
\hline & & & & & & & & Kraubat1 & & & & & & & & \\
\hline 6.74 & 0.070 & 0.079 & 0.050 & 0.100 & 0.116 & 0.599 & 24.47 & 0.023 & 10.07 & 1.04 & 2.21 & 0.118 & 1.336 & 0.135 & 0.120 & 2.24 \\
\hline 8.74 & 0.030 & 0.063 & 0.014 & 0.087 & 0.092 & 0.562 & 34.81 & 0.009 & 9.92 & 0.39 & 1.33 & 0.108 & 1.073 & 0.170 & 0.133 & 1.80 \\
\hline 9.72 & 0.021 & 0.061 & 0.007 & 0.096 & 0.095 & 0.448 & 36.57 & 0.007 & 8.94 & 0.32 & 1.03 & 0.104 & 0.829 & 0.214 & 0.128 & 1.27 \\
\hline 10.73 & 0.043 & 0.059 & 0.029 & 0.103 & 0.100 & 0.408 & 37.05 & 0.006 & 8.34 & 0.35 & 0.89 & 0.101 & 0.693 & 0.247 & 0.122 & 1.05 \\
\hline 11.56 & 0.044 & 0.058 & 0.030 & 0.107 & 0.099 & 0.337 & 36.48 & 0.005 & 7.76 & 0.40 & 0.83 & 0.098 & 0.573 & 0.259 & 0.111 & 0.85 \\
\hline & & & & & & & & bath $+\mathrm{r}$ & & & & & & & & \\
\hline 3.88 & 0.903 & 0.063 & 0.817 & 0.226 & 0.096 & 1.933 & 21.81 & 0.045 & 23.28 & 23.38 & 57.70 & 0.114 & 1.833 & 0.020 & 0.070 & 14.03 \\
\hline 4.23 & 0.688 & 0.059 & 0.652 & 0.497 & 0.096 & 2.302 & 30.36 & 0.036 & 41.72 & 28.38 & 67.22 & 0.108 & 1.508 & 0.010 & 0.089 & 12.61 \\
\hline 4.75 & 0.445 & 0.057 & 0.416 & 0.688 & 0.101 & 1.509 & 32.94 & 0.025 & 46.16 & 22.74 & 55.35 & 0.103 & 1.164 & 0.027 & 0.091 & 9.03 \\
\hline 5.41 & 0.283 & 0.054 & 0.260 & 0.744 & 0.101 & 1.189 & 32.62 & 0.017 & 44.99 & 16.99 & 42.86 & 0.097 & 0.907 & 0.031 & 0.082 & 6.40 \\
\hline 5.85 & 0.226 & 0.053 & 0.207 & 0.729 & 0.101 & 0.966 & 32.70 & 0.014 & 41.76 & 14.50 & 37.52 & 0.097 & 0.828 & 0.040 & 0.078 & 5.41 \\
\hline
\end{tabular}

PK: mineral fertiliser added; metal: metal solution added.

\subsection{Kinetic Modelling}

The modelling of release kinetics from soil can be accomplished by using the measured concentrations versus time, but also by using the accumulated (integrated) concentrations. In case the measured concentrations decrease versus time, a time can be extrapolated, 
when the concentration will reach zero. This means a constant value for the accumulated concentration, such as exhaustion. The corresponding accumulated concentration reached at this time can be regarded as maximum releasable. Although different parameters a and b were obtained from fittings due to different kinetic equations (Table 2), these maximum release concentrations were rather similar.

The integrated curves can be used to model the release mechanism. Fitting parameters are the time to reach the maximum releasable amount, the time to reach it, and the slope and intercept of the fitting curves. The initial release rate is defined just for the linear equation as parameter " $a$ ", but for the Elovich and Weber-Morris equation, it is also " $a$ " for $t=1$, which is close to zero with respect to an observation time of 30 .

Thus, combining values of plant uptake with parameters "a" would yield correlations to an almost initial release rate in the cases of linear, Elovich, and Weber-Morris equations, and combining values with parameters " $b$ " would yield correlations with the intercept of the accumulated release curve, which means the extrapolated EUF release at zero time in the cases of linear and Weber-Morris equations.

\subsection{Connections with Plant Uptake}

From measured concentrations and yield, the amount of metal content present in lettuce plants after the growth period in the respective substrates was calculated and correlated with the four fitting parameters of the kinetic models.

\section{Results}

Root dry mass reached only about $1 / 5$ of leaf dry mass. Additions of PK fertiliser or metal salt solution hardly affected leaf dry masses but lowered uptake of $\mathrm{Pb}$ and increased $\mathrm{Cu}$ and $\mathrm{Zn}$. Metal salt additions increased $\mathrm{Pb}$ and $\mathrm{Ni}$, as expected. Other authors also found that the addition of $\mathrm{P}$ fertiliser lowered the release of $\mathrm{Pb}$ from soil $[17,18]$.

The parameters resulting from the EUF procedure, as well as decreasing, increasing, and constant concentrations versus time are discussed in this section. Partition of the release curves into zones of different rate laws was not possible due to the measurement of only five points.

Decreasing concentrations versus time were observed in $\mathrm{As}, \mathrm{Cd}, \mathrm{Cu}, \mathrm{Li}, \mathrm{Ni}, \mathrm{Pb}, \mathrm{Sr}$, and $\mathrm{Zn}$. This means exhaustion of the releasable fraction and possible extrapolation to obtain a maximum releasable amount and the corresponding time. Conversely, the concentrations of $\mathrm{Al}, \mathrm{Fe}$, and Co increased versus electrolysis time, which may be interpreted as an activation of release by the dissolution of less soluble coatings [6]. Others, such as $\mathrm{Mn}, \mathrm{Sb}$, or V, yielded constant release during the observation period (Table 3).

The linear equation $y=b+a t(a=$ slope, $b=$ intercept $)$ would correspond to the dissolution of a homogenous solid. In this case, fitting was worst in most cases.

The Elovich equation $y=b+a \ln (t)$ is valid if the release rate decreases because of decreasing surface covering. In this work, this was the case for $\mathrm{As}, \mathrm{Cd}$, and $\mathrm{Cu}$, and preferably for $\mathrm{Zn}$. This equation also performed best to model the release and uptake of $\mathrm{K}$ from Chinese red clay soils [19].

The Weber-Morris equation $\mathrm{y}=\mathrm{b}+\mathrm{a} \sqrt{ } \mathrm{t}$ is valid if the transport from reactive surfaces is rate determining; thus, the dissolution is diffusion controlled. The dissolution can be parted in various steps, such as desorption from the solid, diffusion inside the solid, film diffusion, and diffusion within the liquid [20]. The intercept " $b$ " is a diffusion constant and proportional to the interface layer thickness [6,20]. If the intra-particle diffusion is rate controlling, the curves should pass the origin [21]. In this work, the Weber-Morris fit was best in $\mathrm{Pb}$ and $\mathrm{Sr}$ for all samples, and for $\mathrm{Li}$ and $\mathrm{Ni}$ in major cases.

The power equation $\mathrm{y}=\mathrm{b} \cdot \mathrm{t}^{\mathrm{a}}$ or $\ln (\mathrm{y})=\mathrm{b}+\mathrm{a} \ln (\mathrm{t})$ has also been used by some authors to model the release of plant nutrients or dissolution of minerals [19].

In the cases of As and $\mathrm{Cd}$, correlation coefficients between plant uptake and kinetic parameters (Table 4) remained minor 0.45 , in spite of additions of soluble $\mathrm{Cd}$. Cu contents correlated for roots only, at best for the Weber-Morris model. $\mathrm{Ni}$, and, to a lesser extent, $\mathrm{Pb}$, 
correlated positively with the slopes " $a$ ", the intercepts " $b$ ", and the maximum released concentrations, but this was biased by the samples receiving the metal solution.

Table 4. Correlation coefficients between the contents in cropped lettuce and the kinetic parameters obtained from modelling the EUF data.

\begin{tabular}{|c|c|c|c|c|c|c|c|c|c|c|c|c|}
\hline \multirow[b]{2}{*}{ Correlations } & \multicolumn{3}{|c|}{ Time to Reach Max. Conc. } & \multicolumn{3}{|c|}{ Slope a } & \multicolumn{3}{|c|}{ Intercept $\mathbf{b}$} & \multicolumn{3}{|c|}{ Maximum Releasable Concentrations } \\
\hline & Leaf $\mu \mathrm{g}$ & Root $\mu \mathrm{g}$ & Sum $\mu \mathrm{g}$ & Leaf $\mu \mathrm{g}$ & Root $\mu \mathrm{g}$ & Sum $\mu \mathrm{g}$ & Leaf $\mu \mathrm{g}$ & Root $\mu \mathrm{g}$ & Sum $\mu \mathrm{g}$ & Leaf $\mu \mathrm{g}$ & Root $\mu \mathrm{g}$ & Sum $\mu \mathrm{g}$ \\
\hline As & & & & & & & & & & & & \\
\hline Elovich & 0.2134 & 0.0723 & 0.2523 & 0.2792 & -0.3769 & 0.1625 & -0.2916 & 0.3870 & -0.1719 & 0.2799 & -0.3881 & 0.1595 \\
\hline Weber-Morris & 0.1848 & 0.0941 & 0.2289 & 0.2796 & -0.3777 & 0.1626 & -0.3054 & 0.3991 & -0.1820 & 0.2778 & -0.3930 & 0.1558 \\
\hline $\begin{array}{l}\text { Linear } \\
\mathrm{Cd}\end{array}$ & 0.1642 & 0.0968 & 0.2078 & 0.2800 & -0.3782 & 0.1629 & 0.2619 & -0.3601 & 0.1503 & 0.2748 & -0.4001 & 0.1503 \\
\hline Elovich & -0.4708 & -0.2838 & -0.4652 & 0.2163 & 0.4371 & 0.2505 & -0.2018 & -0.4061 & -0.2334 & 0.2095 & 0.4299 & 0.2433 \\
\hline Weber-Morris & -0.4370 & -0.2290 & -0.4277 & 0.2086 & 0.4151 & 0.2407 & -0.1744 & -0.3441 & -0.2009 & 0.2012 & 0.4135 & 0.2338 \\
\hline $\begin{array}{l}\text { Linear } \\
\mathrm{Cu}\end{array}$ & -0.4465 & -0.2328 & -0.4368 & 0.2155 & 0.4370 & 0.2497 & 0.2339 & 0.4721 & 0.2707 & 0.1996 & 0.4253 & 0.2337 \\
\hline Elovich & 0.2296 & $-0.6832 *$ & 0.0922 & 0.1437 & -0.2802 & 0.0937 & 0.0058 & 0.4202 & 0.1092 & 0.2578 & -0.2435 & 0.2314 \\
\hline Weber-Morris & 0.2982 & $-0.8266 * *$ & 0.1345 & 0.1643 & -0.3121 & 0.1091 & -0.4483 & 0.7995 ** & -0.3105 & 0.1800 & -0.4092 & 0.1031 \\
\hline $\begin{array}{l}\text { Linear } \\
\mathrm{Ni}\end{array}$ & 0.3102 & $-0.8229 * *$ & 0.1489 & 0.1746 & -0.3290 & 0.1166 & -0.3638 & 0.6371 * & -0.2548 & 0.1834 & -0.4164 & 0.1051 \\
\hline Elovich & -0.2415 & -0.2803 & -0.2559 & $0.7272 *$ & $0.8545 * *$ & $0.7729 * *$ & $-0.7412 * *$ & $-0.8431^{* *}$ & $-0.7819^{* *}$ & $0.7274 *$ & $0.8492 * *$ & $0.7719 * *$ \\
\hline Weber-Morris & -0.2074 & -0.2984 & -0.2319 & 0.7276 * & $0.8541^{* *}$ & 0.7731 ** & $-0.7463 * *$ & $-0.8324 * *$ & -0.7838 ** & 0.7341 * & 0.8499 ** & 0.7775 ** \\
\hline $\begin{array}{l}\text { Linear } \\
\mathrm{Pb}\end{array}$ & -0.0675 & -0.1540 & -0.0874 & 0.7279 * & 0.8537 ** & 0.7733 ** & $0.6245^{*}$ & $0.8393 * *$ & 0.6858 * & $0.7356 * *$ & 0.8491 ** & $0.7786 * *$ \\
\hline Elovich & 0.3787 & 0.4582 & 0.4743 & 0.6204 * & 0.5133 & 0.6808 * & -0.6275 & -0.5196 & $-0.6888^{*}$ & 0.6314 * & 0.5337 & 0.6975 * \\
\hline Weber-Morris & 0.4863 & 0.4937 & 0.5707 & $0.6208^{*}$ & 0.5140 & $0.6814^{*}$ & $-0.6314^{*}$ & -0.5238 & -0.6935 * & $0.6329 *$ & 0.5351 & 0.6992 * \\
\hline $\begin{array}{l}\text { Linear } \\
\mathrm{Zn}\end{array}$ & 0.5485 & 0.5084 & 0.6240 * & 0.6212 * & 0.5146 & 0.6819 * & 0.5309 & 0.4306 & 0.5791 & 0.6338 * & 0.5357 & 0.7001 * \\
\hline Elovich & 0.3927 & 0.1565 & 0.3615 & 0.8328 ** & $0.7785 * *$ & 0.8488 ** & -0.2999 & -0.2757 & -0.3048 & 0.7588 ** & $0.7069 *$ & $0.7729 * *$ \\
\hline Weber-Morris & 0.4729 & 0.3029 & 0.4564 & 0.8316 ** & 0.7770 ** & $0.8475^{\text {** }}$ & $-0.7877^{* *}$ & -0.6916 * & -0.7946 ** & 0.7996 ** & $0.7584^{* *}$ & 0.8170 ** \\
\hline $\begin{array}{c}\text { Linear } \\
\mathrm{Li}\end{array}$ & $\begin{array}{c}0.5930 \\
\mathrm{Li}\end{array}$ & 0.3597 & 0.5685 & 0.8304 ** & 0.7755 ** & 0.8463 ** & $0.8579 * *$ & $0.8648^{* *}$ & 0.8860 ** & $0.8155 * *$ & 0.7575 ** & $0.8303^{* *}$ \\
\hline Elovich & -0.1197 & -0.3346 & -0.2006 & 0.5613 & 0.4747 & $0.6433 *$ & -0.5397 & -0.4980 & -0.6299 * & 0.5900 & 0.4108 & 0.6523 * \\
\hline Weber-Morris & 0.0549 & -0.0205 & 0.0447 & 0.5640 & 0.4757 & $0.6461 *$ & 0.5165 & -0.3511 & 0.3780 & 0.6633 * & 0.2855 & 0.6854 * \\
\hline $\begin{array}{l}\text { Linear } \\
\text { Sr }\end{array}$ & $\begin{array}{l}-0.3865 \\
\mathrm{Sr}\end{array}$ & -0.4020 & -0.4634 & 0.5511 & 0.4694 & 0.6325 * & 0.0585 & -0.3236 & -0.0343 & 0.3898 & -0.0718 & 0.3377 \\
\hline Elovich & -0.0994 & 0.0188 & -0.0920 & 0.0443 & 0.3374 & 0.0709 & -0.3794 & -0.4329 & -0.3955 & -0.0200 & 0.2541 & 0.0048 \\
\hline Weber-Morris & 0.4391 & $0.6361 *$ & 0.4694 & 0.3255 & 0.3134 & 0.3344 & -0.5136 & -0.4951 & -0.5276 & 0.4156 & 0.4522 & 0.4313 \\
\hline Linear & 0.6141 * & 0.5901 & $0.6306^{*}$ & 0.2541 & 0.3038 & 0.2661 & -0.1086 & -0.0917 & -0.1104 & 0.3961 & 0.4504 & 0.4133 \\
\hline
\end{tabular}

Parameters $\mathrm{a}$ and $\mathrm{b}$ refer to the respective definitions given in Table $2 .{ }^{*}$ means $p<0.05$ or $>95 \%$ of confidence, ** means $p<0.01$ or $>99 \%$ of confidence. Correlation coefficients $>0.5$ have been marked bold.

Zn correlated strongly positively with the slopes " $a$ ", and a little less with the intercept " $b$ ", the maximum release, and the corresponding time of either model. The linear approximation could not fit the curvatures and correlated slightly negatively with its intercept " $\mathrm{b}$ ".

Among the cations of low physiological activity for green plants (e.g., Li, $\mathrm{Sr}$ ), greenplant $\mathrm{Li}$ increased with increasing slopes and maximum release, whereas the time to reach this release was not relevant. For Sr, however, linear modelling proved best, and there was a good correlation with the releasable amount and its corresponding time.

Results of all correlation coefficients are presented in Table 4, and the corresponding probability values $p$ are listed in Table 5 . Correlations with probability values $<0.05$ for the null hypothesis ( $>95 \%$ confidence level of significance), calculated as 2 -sided $p$ from the SPSS statistics program (version 25), are marked ${ }^{*}$, and those $<0.01$ are marked ${ }^{* *}$ in Table 4.

Table 5. Probability values between the contents in cropped lettuce and the kinetic parameters obtained from modelling the EUF data.

\begin{tabular}{|c|c|c|c|c|c|c|c|c|c|c|c|c|}
\hline \multirow[b]{2}{*}{ Probability } & \multicolumn{3}{|c|}{ Time to Reach Max. Conc. } & \multicolumn{3}{|c|}{ Slope a } & \multicolumn{3}{|c|}{ Intercept b } & \multicolumn{3}{|c|}{$\begin{array}{l}\text { Maximum Releasable } \\
\text { Concentrations }\end{array}$} \\
\hline & Leaf $\mu \mathrm{g}$ & Root $\mu \mathrm{g}$ & Sum $\mu \mathrm{g}$ & Leaf $\mu \mathrm{g}$ & Root $\mu \mathrm{g}$ & Sum $\mu \mathrm{g}$ & Leaf $\mu \mathrm{g}$ & Root $\mu \mathrm{g}$ & Sum $\mu \mathrm{g}$ & Leaf $\mu \mathrm{g}$ & Root $\mu \mathrm{g}$ & Sum $\mu \mathrm{g}$ \\
\hline As & & & & & & & & & & & & \\
\hline Elovich & 0.501 & 0.288 & 0.293 & 0.406 & 0.253 & 0.633 & 0.384 & 0.24 & 0.613 & 0.403 & 0.238 & 0.638 \\
\hline Weber-Morris & 0.513 & 0.301 & 0.307 & 0.405 & 0.252 & 0.633 & 0.361 & 0.224 & 0.592 & 0.409 & 0.231 & 0.649 \\
\hline $\begin{array}{l}\text { Linear } \\
\text { Cd }\end{array}$ & 0.501 & 0.299 & 0.297 & 0.404 & 0.251 & 0.632 & 0.437 & 0.277 & 0.659 & 0.413 & 0.224 & 0.658 \\
\hline Elovich & 0.144 & 0.398 & 0.149 & 0.523 & 0.179 & 0.457 & 0.552 & 0.216 & 0.489 & 0.539 & 0.187 & 0.472 \\
\hline Weber-Morris & 0.179 & 0.498 & 0.189 & 0.538 & 0.205 & 0.475 & 0.608 & 0.300 & 0.553 & 0.553 & 0.206 & 0.488 \\
\hline $\begin{array}{l}\text { Linear } \\
\mathrm{Cu}\end{array}$ & 0.169 & 0.491 & 0.179 & 0.524 & 0.179 & 0.458 & 0.489 & 0.143 & 0.420 & 0.554 & 0.191 & 0.486 \\
\hline Elovich & 0.497 & $0.020 *$ & 0.788 & 0.673 & 0.404 & 0.782 & 0.987 & 0.198 & 0.749 & 0.444 & 0.470 & 0.493 \\
\hline Weber-Morris & 0.373 & $0.002 * *$ & 0.693 & 0.629 & 0.350 & 0.749 & 0.167 & $0.003^{* *}$ & 0.352 & 0.597 & 0.211 & 0.763 \\
\hline Linear & 0.353 & $0.002 * *$ & 0.662 & 0.608 & 0.323 & 0.733 & 0.271 & 0.035 * & 0.449 & 0.589 & 0.203 & 0.758 \\
\hline
\end{tabular}


Table 5. Cont.

\begin{tabular}{|c|c|c|c|c|c|c|c|c|c|c|c|c|}
\hline \multirow[b]{2}{*}{ Probability } & \multicolumn{3}{|c|}{ Time to Reach Max. Conc. } & \multicolumn{3}{|c|}{ Slope a } & \multicolumn{3}{|c|}{ Intercept b } & \multicolumn{3}{|c|}{$\begin{array}{l}\text { Maximum Releasable } \\
\text { Concentrations }\end{array}$} \\
\hline & Leaf $\mu \mathrm{g}$ & Root $\mu \mathrm{g}$ & Sum $\mu \mathrm{g}$ & Leaf $\mu \mathrm{g}$ & Root $\mu \mathrm{g}$ & Sum $\mu \mathrm{g}$ & Leaf $\mu \mathrm{g}$ & Root $\mu \mathrm{g}$ & Sum $\mu \mathrm{g}$ & Leaf $\mu \mathrm{g}$ & Root $\mu \mathrm{g}$ & Sum $\mu \mathrm{g}$ \\
\hline $\mathrm{Ni}$ & & & & & & & & & & & & \\
\hline Elovich & 0.474 & 0.404 & 0.448 & $0.011 *$ & $0.001 * *$ & $0.005^{* *}$ & $0.009 * *$ & $0.001 * *$ & $0.004 * *$ & $0.011 *$ & $0.001 * *$ & $0.005 * *$ \\
\hline Weber-Morris & 0.541 & 0.373 & 0.498 & $0.011 *$ & $0.001 * *$ & $0.005^{* *}$ & $0.008^{* *}$ & $0.001 * *$ & $0.004 * *$ & $0.010 *$ & $0.001 * *$ & $0.005^{* *}$ \\
\hline $\begin{array}{l}\text { Linear } \\
\mathrm{Pb}\end{array}$ & 0.844 & 0.651 & 0.798 & 0.011 * & $0.001 * *$ & $0.005^{* *}$ & $0.040 *$ & $0.001 *$ & $0.020 *$ & $0.010^{* *}$ & $0.001^{* *}$ & $0.005^{* *}$ \\
\hline Elovich & 0.251 & 0.156 & 0.140 & $0.042 *$ & 0.106 & $0.021 *$ & 0.039 * & 0.101 & $0.019 *$ & $0.037^{*}$ & 0.091 & $0.017 *$ \\
\hline Weber-Morris & 0.129 & 0.123 & 0.067 & $0.042 *$ & 0.106 & $0.021 *$ & 0.037 * & 0.098 & $0.018 *$ & $0.037 *$ & 0.090 & 0.017 * \\
\hline $\begin{array}{l}\text { Linear } \\
\text { Zn }\end{array}$ & 0.081 & 0.110 & $0.040 *$ & $0.041 *$ & 0.105 & $0.021 *$ & 0.093 & 0.186 & 0.062 & $0.036^{*}$ & 0.089 & $0.016^{*}$ \\
\hline Elovich & 0.231 & 0.647 & 0.273 & $0.001 * *$ & $0.005^{* *}$ & $0.001 * *$ & 0.374 & 0.416 & 0.361 & $0.007^{* *}$ & $0.014 *$ & $0.005^{* *}$ \\
\hline Weber-Morris & 0.141 & 0.364 & 0.157 & $0.001 * *$ & $0.005 * *$ & $0.001 * *$ & $0.004^{* *}$ & 0.018 * & 0.003 ** & 0.003 ** & $0.007^{* *}$ & 0.002 ** \\
\hline $\begin{array}{l}\text { Linear } \\
\mathrm{Li}\end{array}$ & 0.054 & 0.275 & 0.067 & 0.002 ** & $0.005^{* *}$ & 0.001 ** & $0.001 * *$ & 0.001 ** & $0.000 * *$ & 0.002 ** & $0.007^{* *}$ & 0.002 ** \\
\hline Elovich & 0.722 & 0.316 & 0.555 & 0.073 & 0.139 & $0.033 *$ & 0.087 & 0.119 & 0.038 & 0.054 & 0.183 & $0.027 *$ \\
\hline Weber-Morris & 0.874 & 0.956 & 0.894 & 0.071 & 0.137 & $0.032 *$ & 0.103 & 0.298 & 0.250 & 0.029 * & 0.360 & 0.021 * \\
\hline $\begin{array}{l}\text { Linear } \\
\text { Sr }\end{array}$ & 0.238 & 0.220 & 0.151 & 0.079 & 0.144 & 0.037 * & 0.860 & 0.329 & 0.922 & 0.229 & 0.867 & 0.297 \\
\hline Elovich & 0.695 & 0.749 & 0.730 & 0.897 & 0.339 & 0.838 & 0.250 & 0.190 & 0.229 & 0.954 & 0.451 & 0.991 \\
\hline Weber-Morris & 0.177 & $0.032 *$ & 0.145 & 0.329 & 0.350 & 0.315 & 0.106 & 0.121 & 0.096 & 0.203 & 0.163 & 0.185 \\
\hline Linear & $0.045^{*}$ & 0.057 & 0.037 & 0.452 & 0.374 & 0.429 & 0.750 & 0.774 & 0.746 & 0.229 & 0.165 & 0.207 \\
\hline
\end{tabular}

Parameters $\mathrm{a}$ and $\mathrm{b}$ refer to the respective definitions given in Table $2 .{ }^{*}$ means $p<0.05$ or $>95 \%$ of confidence, ${ }^{* *}$ means $p<0.01$ or $>99 \%$ of confidence.

\section{Discussion}

In this work, $0.002 \mathrm{M}$ DTPA (di-ethylenetriaminepenta-acetic acid, $\mathrm{C}_{14} \mathrm{H}_{23} \mathrm{~N}_{3} \mathrm{O}_{10}$, known as Merck Titriplex V) was used as an electrolyte in the EUF procedure at proper soil pH levels. DTPA is no component of root exudates, but no electrochemical reactions are expectable. Due to its high solubility in water, the free acid can be used, contrary to EDTA, which has to be taken as the $\mathrm{Na}_{2}$-salt. DTPA is, therefore, more convenient for the ICP-OES measurement and prevents ion exchanges with Na. Compared with EDTA, the DTPA is forming stronger complexes with most metal cations, but which is only relevant at $\mathrm{pH}>7$, because DTPA is also a weaker acid [22].

Extractions with DTPA, buffered with tri-ethanolamine at $\mathrm{pH}$ 7.3, have been already used in 1976 to monitor plant-available $\mathrm{Zn}, \mathrm{Fe}, \mathrm{Mn}$, and $\mathrm{Cu}$ concentrations in Colorado soils. After rapid extraction with the first $5 \mathrm{~min}$, the dissolution equilibrium had not been reached after $2 \mathrm{~h}$, but there was a strong correlation with the amounts obtained after $30 \mathrm{~min}$, thus shortening the extraction time $[23,24]$.

Doubling the voltage used in the EUF method led to similar results; the EUF conditions thus do not seem to be critical [19]. Additionally, though the extrapolated time to reach zero release differed between the models to some extent, the corresponding releasable concentrations calculated from the cumulative curves were rather similar, regardless of the kinetic model used.

From the metalliferous soil samples used, uptake of $\mathrm{Zn}, \mathrm{Li}$, and $\mathrm{Sr}$ could be well correlated with parameters of kinetic models from the release in $0.002 \mathrm{M}$ DTPA obtained via the EUF method, while this was not the case for As, Cd, and leafy Cu (Table 4). In cases in which the desorption from the soil matrix to the soil solution is slower than the uptake by plant roots, the kinetics of soil desorption directly reflect the soil-plant transfer. This assumes that plant uptake is faster than dissolution $[25,26]$. If the plant uptake does not correspond with kinetic parameters, this means either that the plant does not take just what is released, but what it needs or rejects, or that the use of another electrolyte closer to physiological conditions might be more suitable. Surprisingly, the significance of correlations between plant uptake and kinetic parameters was similar, irrespective of the kinetic model used. Fitting all four parameters versus plant uptake into one equation by partial correlation would be more conclusive, but this feature was not contained in the statistics program which the author could use. 


\section{Conclusions}

Most plant physiologists [1,2] assume that plant uptake of nutrients and metals is governed by respective amounts released into the soil solution. The This implicates that dissolution is rate determining but not the needs and the uptake mechanisms of the plant itself. Thus, plant-available fractions in soils were defined via selective dissolution obtained by various extractants, reaching some equilibrium by shaking. However, because plant uptake is a kinetic process, the specific point of this pilot study was to investigate the soil-to-plant transfer via the study of dissolution kinetics rather than a static process, as in the EUF procedure, the backward reaction is prevented by the removal of released items in the electric field, and the forward reactions become accessible.

Within this pilot study, only four types of soil could be used, but the range of concentrations and availabilities was amplified by adding $\mathrm{PK}$ fertiliser or a $\mathrm{Cd}-\mathrm{Ni}-\mathrm{Pb}$ solution to each of them. As a result, the uptake of $\mathrm{Zn}, \mathrm{Li}$, and also $\mathrm{Ni}$ and $\mathrm{Pb}$ into salad plants, and for roots also $\mathrm{Cu}$, yielded significant correlations with kinetic dissolution parameters, whereas $\mathrm{Cd}$ and As did not. Thus, the plant does not necessarily absorb all that is released from the soil. Best-fit comparisons show that the optimum fit was obtained due to the released element but not due to the type of soil used (Table 4).

This should encourage further studies to use the EUF technique for other electrolytes and test plants, in order to optimise availability predictions, and on the other hand, to find nutrients and trace elements in combination with crops, the uptake of which does not depend merely on solubility. The method is quick-within half an hour, five solutions were obtained, and evaluations of the ICP-OES data might be programmed to obtain automatisation for routine use.

Funding: This research received no external funding. The soil samples were submitted by courtesy from the Project ReSoil, investigating metalliferous soils from the Province of Styria/Austria, and funded by the Austrian Research Promotion Agency.

Institutional Review Board Statement: Not applicable.

Informed Consent Statement: Not applicable.

Data Availability Statement: Additional data available from the author upon request.

Acknowledgments: The EUF procedures for soil samples were performed at the Justut-Liebig Laboratory at Rain (Bavaria, Germany) of the Südzucker AG, by A. Jelecevic, with the help of the local staff, to whom the author is greatly indebted.

Conflicts of Interest: The author has no conflict of interest.

\section{References}

1. Marschner, H. Mineral Nutrition of Higher Plants, 2nd ed.; Academic Press: Cambridge, MA, USA, 1995.

2. Wissemeier, A.; Olfs, H.W. (Eds.) Den Nährstoffen Auf der Spur; Erling Verlag: Clenze, Germany, 2019; p. 288. ISBN 978-3-86263-118-6.

3. Jelecevic, A.; Wellacher, M.; Sager, M.; Liebhard, P. Heavy metals in soils from selected locations in Styria. Wasser Und Abfall 2018, 5, 25-31. (In German) [CrossRef]

4. Sager, M. Chemical speciation and environmental mobility of heavy metals in sediments and soils. In Hazardous Metals in the Environment; Stoeppler, M., Ed.; Elsevier Science Publishers: Amsterdam, The Netherlands, 1992; pp. $133-175$.

5. Sager, M. Metals in the environment, mobility, bioaccessibility and contamination. Carpathian J. Earth Environ. Sci. 2020, 15, 47-70. [CrossRef]

6. Grilli, E.; Ganga, A.; Salvestrini, S. Modelling the kinetics of elements release from a zeolitic rich tuff. Environments 2020, 7, 41. [CrossRef]

7. Berner, R.A. Rate control of mineral dissolution under earth surface conditions. Am. J. Sci. 1978, 278, 1235-1252. [CrossRef]

8. Pirkl, H.; Schedl, A.; Pfleiderer, S. Geochemischer Atlas von Österreich-Bundesweite Bach- und Flusssedimentgeochemie. Arch. Für Lagerstättenforschung 2015, 28, 288.

9. ÖNORM L 1056 Sampling of Grassland (Including Parks and Green Areas), 1 July 2004. Available online: https: //shop.austrian-standards.at/action/en/public/details/180359/OENORM_L_1056_2004_07_01;jsessionid=A59B4033BB796 D779C2AD043B70A7020 (accessed on 26 December 2021). 
10. ÖNORM EN 15933: Sludge, Treated Biowaste and Soil-Determination of pH, 1 October 2012. Available online: https://shop.austrian-standards.at/action/de/public/details/445999/OENORM_EN_15933_2012_10_01;jsessionid=1FAAF9 37AC2C2224FE4402967B6E9B36 (accessed on 26 December 2021).

11. ÖNORM L 1061-2 Physical Soil Investigations-Determination of Grain Size Distributions of Mineral Soils. Part 2 Fine Soils, 1 February 2002. Available online: https:/ / www.bdb.at/Service/NormenDetail?id=651037 (accessed on 26 December 2021).

12. ÖNORM L 1080 Chemical Analysis of Soil; Determination of Humus by Dry Combustion of Carbon, 1 January 1989. Available online: http://www.ydylstandards.org.cn/static/down/pdf/ONORM\%20L\%201080-1999_5625.pdf (accessed on 26 December 2021).

13. Sager, M.; Chon, H.T.; Lee, S.Y. Growth of lettuce (lactuca sativa) at mine tailings from Shiheung/ Korea, and the effect of added organic complexants on metal mobilities. Ecol. Chem. Eng. 2010, 17, 177-197.

14. Németh, K. Electro-ultrafiltration of aqueous soil suspension with simultaneously varying temperature and voltage. Plant Soil 1982, 64, 7-23. [CrossRef]

15. Nemeth, K. Recent advances in EUF research (1980-1983). Plant Soil 1985, 83, 1-19. [CrossRef]

16. Horn, D. Determination of micronutrients and heavy metals in soils using electro-ultrafiltration (EUF) technique by addition of DTPA (in German). J. Plant Nutr. Soil Sci. 2006, 169, 83-86. [CrossRef]

17. Ma, Q.Y.; Traina, S.J.; Logan, T.J.; Ryan, J.A. Effects of aqueous $\mathrm{Al}, \mathrm{Cd}, \mathrm{Cu}, \mathrm{Fe}(\mathrm{II}), \mathrm{Ni}$, and $\mathrm{Zn}$ on $\mathrm{Pb}$ immobilization by hydroxyapatite. Environ. Sci. Technol. 1994, 28, 1219-1228. [CrossRef] [PubMed]

18. Hafsteinsdottir, E.G.; Camenzuli, D.; Rocavert, A.L.; Walworth, J.; Gore, D.B. Chemical immobilization of metals and metalloids by phosphates. Appl. Geochem. 2015, 59, 47-62. [CrossRef]

19. Lü, X.-N.; Xu, J.-M.; Ma, W.-Z.; Lu, Y.-F. Comparison of seven kinetic equations for K-release and application of kinetic parameters. Pedosphere 2007, 17, 124-129. [CrossRef]

20. Kumar, E.; Bhatnagar, A.; Ji, M.; Jung, W.; Lee, S.-H.; Kim, S.-J.; Lee, G.-H.; Song, H.-C.; Choi, J.-Y.; Yang, J.-S.; et al. Defluoridation from aqueous solutions by granular ferric hydroxide. Water Res. 2009, 43, 490-498. [CrossRef] [PubMed]

21. Daifullah, A.A.M.; Yakout, S.M.; Elreefy, S.A. Adsorption of fluoride in aqueous solutions using $\mathrm{KMnO}_{4}$-modified activated carbon derived from steam pyrolysis of rice straw. J. Hazard. Mat. 2007, 147, 633-643. [CrossRef] [PubMed]

22. Anderegg, G.; Nägeli, P.; Müller, F.; Schwarzenbach, G.; Komplexone, X. Diäthylentriamin-pentaessigsäure (DTPA). Helv. Chim. Acta 1959, 42, 827-836. [CrossRef]

23. Soltanpour, P.N.; Khan, A.; Lindsay, W.L. Factors affecting DTPA-extractable Zn, Fe, Mn, and Cu from soils. Commun. Soil Sci. Plant Anal. 1976, 7, 797-821. [CrossRef]

24. Lindsay, W.L.; Norvell, W.A. Development of a DTPA Soil Test for Zinc, Iron, Manganese, and Copper. Soil Sci. Am. J. 1978, 42, 421-428. [CrossRef]

25. Dang, Y.P.; Dalal, R.C.; Edwards, D.G.; Tiller, K.G. Kinetics of zinc desorption from vertisols. Soil Sci. Soc. Am. J. 1994, 58, 1392-1399. [CrossRef]

26. Motlagh, M.B. Kinetics and mechanism of copper release from selected agricultural calcareous soils of northern Iran. Soil Res. 2012, 50, 312-319. [CrossRef] 